\title{
Ki67 index is an independent prognostic factor in epithelioid but not in non-epithelioid malignant pleural mesothelioma: a multicenter study
}

B Ghanim ${ }^{1,2,14}$, T Klikovits ${ }^{1,14}$, M A Hoda ${ }^{1,2}$, G Lang ${ }^{1,3}$, I Szirtes ${ }^{4}$, U Setinek ${ }^{5}$, A Rozsas ${ }^{1,6}$, F Renyi-Vamos ${ }^{1,7}$, V Laszlo ${ }^{1}$, M Grusch ${ }^{2}$, M Filipits ${ }^{2}$, A Scheed ${ }^{1}$, M Jakopovic ${ }^{8}$, M Samarzija ${ }^{8}$, L Brcic ${ }^{9}$, D Stancic-Rokotov ${ }^{10}$, I Kern ${ }^{11}$, A Rozman ${ }^{11}$, G Dekan ${ }^{12}$, W Klepetko ${ }^{1}$, W Berger ${ }^{2}$, T Glasz ${ }^{4,6}$, B Dome ${ }^{1,3,6,7,15}$ and B Hegedus ${ }^{\star}, 1,13,15$

${ }^{1}$ Department of Surgery, Comprehensive Cancer Center, Division of Thoracic Surgery, Medical University of Vienna, Währinger Gürtel 18-20, 1090 Vienna, Austria; ${ }^{2}$ Institute of Cancer Research and Comprehensive Cancer Center, Medical University of Vienna, 1090 Vienna, Austria; ${ }^{3}$ Department of Thoracic Surgery, Semmelweis University, 1085 Budapest, Hungary; ${ }^{4}$ 2nd Institute of Pathology, Semmelweis University, 1085 Budapest, Hungary; ${ }^{5}$ Division of Pathology, Otto Wagner Hospital, 1145 Vienna Austria; ${ }^{6}$ National Koranyi Institute of TB and Pulmonology, 1121 Budapest, Hungary; ${ }^{7}$ National Institute of Oncology, 1525 Budapest, Hungary; ${ }^{8}$ Department for Respiratory Diseases Jordanovac, School of Medicine, University of Zagreb, University Hospital Center, 10000 Zagreb, Croatia; ${ }^{9}$ Institute of Pathology, School of Medicine, University of Zagreb, 10000 Zagreb, Croatia; ${ }^{10}$ Department of Thoracic Surgery, University of Zagreb, 10000 Zagreb, Croatia; ${ }^{11}$ University Clinic of Respiratory and Allergic Diseases, 4204 Golnik, Slovenia; ${ }^{12}$ Department of Pathology, Medical University of Vienna, 1090 Vienna, Austria and ${ }^{13}$ MTA-SE Molecular Oncology Research Group, Hungarian Academy of Sciences, 1091 Budapest, Hungary

Background: Estimating the prognosis in malignant pleural mesothelioma (MPM) remains challenging. Thus, the prognostic relevance of Ki67 was studied in MPM.

Methods: Ki67 index was determined in a test cohort of 187 cases from three centres. The percentage of Ki67-positive tumour cells was correlated with clinical variables and overall survival (OS). The prognostic power of Ki67 index was compared with other prognostic factors and re-evaluated in an independent cohort $(n=98)$.

Results: Patients with Ki67 higher than median ( $>15 \%)$ had significantly $(P<0.001)$ shorter median OS $(7.5$ months) than those with low Ki67 (19.1 months). After multivariate survival analyses, Ki67 proved to be-beside histology and treatment—an independent prognostic marker in MPM (hazard ratio (HR): 2.1, $P<0.001$ ). Interestingly, Ki67 was prognostic exclusively in epithelioid $(P<0.001)$ but not in non-epithelioid subtype. Furthermore, Ki67 index was significantly lower in post-chemotherapy samples when compared with chemo-naive cases. The prognostic power was comparable to other recently published prognostic factors (CRP, fibrinogen, neutrophil-to-leukocyte ratio (NLR) and nuclear grading score) and was recapitulated in the validation cohort $(P=0.048)$.

Conclusion: This multicentre study demonstrates that Ki67 is an independent and reproducible prognostic factor in epithelioid but not in non-epithelioid MPM and suggests that induction chemotherapy decreases the proliferative capacity of MPM.

\footnotetext{
*Correspondence: Dr B Hegedus; E-mail: balazs.hegedus@meduniwien.ac.at

${ }^{14}$ These authors contributed equally to the study.

${ }^{15}$ These authors are co-senior authors of this study.
}

Revised 19 December 2014; accepted 25 December 2014; published online 29 January 2015

(C) 2015 Cancer Research UK. All rights reserved 0007 - 0920/15 
Malignant pleural mesothelioma (MPM) is a devastating and often asbestos exposure-associated malignancy with still rising incidence worldwide. Aggressive biological behaviour, therapy resistance and the lack of approved screening methods for early detection result in a median overall survival (OS) of 9 to 17 months only (Tsao et al, 2009). The diagnosis of MPM can be rather challenging and several diagnostic immunohistochemical markers might be necessary to confirm the diagnosis of MPM including WT-1, cytokeratin CK5/6, calretinin, EMA, podoplanin and mesothelin (Scherpereel et al, 2010). As differential diagnostic markers, the detection of CEA, TTF-1, CD15, MOC-31, B72.3 or Ber-Ep4 argues against the diagnosis of MPM (Scherpereel et al, 2010; Husain et al, 2013). However, with regard to prognostic biomarkers, no established immunohistochemical marker exists at the moment to routinely assess prognosis in MPM. Currently, histological subtype is the only established pathological tumour characteristic to routinely estimate the prognosis of MPM patients (Sandeck et al, 2010; Scherpereel et al, 2010).

$\mathrm{Ki} 67$ is a nuclear protein that is detectable in every phase of the cell cycle of proliferating cells but is absent in $\mathrm{G}_{0}$ cells (Gerdes et al, 1984; Yerushalmi et al, 2010). It is therefore widely utilised as a proliferation marker in tumour specimens (Yerushalmi et al, 2010). Ki67 index has been reported to be prognostic in a number of thoracic malignancies including small cell and non-small cell lung cancer (Dingemans et al, 1999; Scagliotti et al, 1993). The mitotic index-reflected by Ki67-is also a diagnostic tool for the identification of pre-neoplastic bronchial lesions and used for tumour classification in neuroendocrine tumours of the lung (Meert et al, 2004; Skov et al, 2010). Ki67 staining on cytological preparations from pleural effusions has been studied as a potential diagnostic marker to discriminate between reactive mesothelial cells and mesothelioma (Hasteh et al, 2010). Ki67 was found to be prognostic in peritoneal mesothelioma (Baratti et al, 2013; Pillai et al, 2013). In MPM patients with short-term and long-term OS, the mitotic index (as determined by the MIB-1 antibody) was reported to differ significantly (Comin et al, 2000; Bongiovanni et al, 2001; Leonardo et al, 2001). Furthermore, Ki67 has been studied in two retrospective patient cohorts previously (Kahlos et al, 2000; Beer, 2001). In a recent study, a nuclear grading score was introduced and was found to be prognostic in epithelioid MPM. This score includes mitotic index that was alone a significant predictor of survival (cutoff: $10 \%$ positive tumour cells) in this tissue microarray analysis (Kadota et al, 2012). In addition, Bitanihirwe et al (2014) found a prognostic value of Ki67 in treatment-naïve MPM patients as well as in patients after induction therapy using tissue microarrays. However, Ki67 is still not an established tool to estimate prognosis in MPM and validation studies showing an independent and reproducible prognostic power are needed before Ki67 can be introduced as a reliable biomarker in MPM. In the present study, we investigated whether Ki67 index measured on whole slides after immunohistochemistry staining is an independent and, furthermore, reproducible prognostic factor in a large international cohort consisting of 187 patients from three different centres and, moreover, in an additional independent validation cohort of 98 patients from yet another centre, adding up to a total of 285 MPM cases.

\section{PATIENTS AND METHODS}

Patients. The test cohort consisted of 187 patients from three centres. In Croatia (CRO), 91 patients were included by the University of Zagreb, School of Medicine, Department for Respiratory Diseases Jordanovac. The Hungarian cohort (HUN) consisted of 42 cases from the National Koranyi Institute of TB and Pulmonology, Budapest. About 54 cases were included in Austria
(AUT) at the Division of Thoracic Surgery, Medical University of Vienna. In addition, 98 patients from the Department for Pulmonology, University Clinic Golnik in Slovenia (SLO) were analysed as an independent validation cohort. All 285 MPM patients were referred to one of the four institutions between 1994 and 2012. Inclusion criteria were a reliable clinical follow-up and available representative paraffin-embedded tumour specimen. In all analysed patients, MPM diagnosis was histologically proven during clinical routine work-up. The IMIG staging system (Rusch, 1995) was used for clinical and pathological tumour staging. All tumour samples were collected either during surgery $(n=71)$ or as part of diagnostic procedures. Clinical data and tumour blocks were retrospectively collected for all cases according to the corresponding local ethic committees.

The CRO cohort (91 patients) involved 85 epithelioid and 6 non-epithelioid MPMs. The majority of patients was treated by chemo and/or radiotherapy $(n=46)$, followed by best supportive care in 29 patients and macroscopic radical surgery alone $(n=6)$ or within a multimodality therapy protocol $(n=6)$. In 4 patients, information about the applied treatment was unavailable in the medical records. In the HUN cohort (42 cases), only three patients received surgery within a multimodality treatment protocol and two additional patients were treated by surgery alone. The epithelioid morphology was the most frequent $(n=33)$ subtype followed by sarcomatoid $(n=5)$ and biphasic $(n=4)$ MPM.

In the AUT cohort, all 54 patients underwent macroscopic complete resection. Extrapleural pneumonectomy was performed in the majority of cases $(n=52)$, as previously described (Aigner et al, 2008). One patient underwent pleurectomy/decortication as defined by Sugarbaker and Wolf (2010), and one additional patient received macroscopic radical local tumour resection followed by adjuvant radiotherapy. All tumour samples were collected during surgery. About 29 patients (53.7\%) received induction chemotherapy before surgery and 23 patients $(42.6 \%)$ were treated with adjuvant therapy after resection (radiation: $n=10$, chemotherapy: $n=8$, chemo-radiation: $n=5$ ), resulting in 36 patients receiving a combination of surgery with adjuvant and/or neoadjuvant treatment (multimodality treatment group). About 18 patients were treated with surgery alone (surgery-alone group). The most frequent histological subtype was the epithelioid $(n=38)$ followed by the biphasic $(n=16)$. No patient in the AUT cohort had sarcomatoid MPM, due to the fact that this histology is a controversial candidate for macroscopic radical tumour resection (Stahel et al, 2010). The majority of all AUT patients (57.4\%) had pathologically classified late stage disease according to the postoperative report (stage III and IV; $n=31$ ).

About 98 SLO patients were analysed as an independent validation cohort to re-evaluate $\mathrm{Ki} 67$ as a reproducible prognostic marker. For the SLO cohort, we used the median Ki67 expression as a cutoff as later described because the tumour samples were scored by an independent pathologist different than the one in the test cohort. Also in this cohort, the epithelioid subtype was the most frequent one $(n=77)$ followed by the biphasic $(n=15)$ and the sarcomatoid $(n=6)$.

Tumour samples. All 285 histological specimens were fixed in formalin and embedded in paraffin (FFPE). One $4-\mu \mathrm{m}$ section from a representative, tumour-rich FFPE block was stained by haematoxylin/eosin to confirm and locate malignant areas and consecutive sections were used for Ki67 immunohistochemistry. Sections were deparaffinised with xylene and rehydrated with decreasing alcohol concentrations. After heat-induced epitope retrieval in citrate buffer $\left(10 \mathrm{mmoll}^{-1}, \mathrm{pH}\right.$ 6.0), slides were incubated at room temperature with the Ki67 antibody (monoclonal mouse antibody, Dako Cytomation (Dako, Glostrup, Denmark), clone MIB-1, dilution $1: 100$, incubation time: $30 \mathrm{~min}$ ). Antibody binding was detected by means of the 
UltraVision LP detection system (Lab Vision Corporation, Fremont, CA, USA) according to the manufacturer's recommendations. Colour development was achieved by 3-3-diaminobenzidine. Finally, all analysed slides were counterstained with Mayer's haematoxylin.

Ki67 scoring. Evaluation of the staining was independently performed by one pathologist (US) in the test cohort and by another independent pathologist (IS) in the validation cohort, both had no information about the clinical history of cases and only received anonymized slides for evaluation and scoring. Both pathologists scored the HUN cohort to test the inter-observer reproducibility. Counting was limited to proven tumour tissue and only nuclear staining was scored as positive. First, the whole tissue section was reviewed at low magnification to identify the regions with the highest viable tumour cell content. The hot-spot approach was used to measure areas with the highest proliferative activity. Whenever possible, up to 10 high-power fields were analysed and at least 500 tumour cells were counted to calculate the mean percentage of Ki67-positive tumour cells per analysed sample.

Nuclear grading score was determined by a board certified pathologist (IS) as previously described by Kadota et al (2012). First, the area with the highest degree of atypia was investigated. The nuclear size and nuclear irregularity was graded as 1 for mild atypia (uniform nuclei), 2 for moderate atypia (intermediate size nuclei and some irregularity in shape), 3 for severe atypia (prominent enlarged nuclei). Next, mitotic count was estimated per 10 high-power fields and the patients were allocated to group 1 for low mitotic count, 2 for intermediate mitotic count and 3 for high mitotic count. Finally, nuclear grading score was calculated as the sum of nuclear atypia and mitotic count (ranging from 2 to 6). The nuclear grading score was dichotomized into the low $(2,3$ and 4$)$ and high (5 and 6) groups for survival analyses. The nuclear grading was performed for 59 epithelioid MPM patients where all other prognostic parameters were available.

Statistical analyses. Metric data is always given as median and corresponding range, or, in case of survival, as median and corresponding $95 \%$ confidence interval (CI) if not otherwise indicated. Overall survival was defined as time between MPM diagnosis and death or, in censored patients, diagnosis and last follow-up date. Survival was analysed by the Kaplan-Meier method and log rank test or by the Cox-regression model to calculate hazard ratios (HRs) and corresponding CIs. In the test cohort, patients were divided into high and low Ki67 index groups by the median (15\%). Accordingly, 92 patients were allocated to the high $(>15 \%)$ and 95 patients to the low Ki67 index group ( $\leqslant 15 \%)$. Since the validation cohort was scored by a different independent pathologist, we again used median Ki67 expression of the validation group (22\%) for dichotomizing into the low $(n=52)$ and high Ki67 $(n=46)$ expressing groups. One-way ANOVA and unpaired $t$-tests were used to compare the mean of parametric distributed metric data between than two groups and more two groups, respectively. Mann-Whitney $U$-test was used for nonparametric distributed metric data. $\chi^{2}$-test was performed for analysing the association between categorical factors. The correlation of metric data was analysed by Pearson's correlation coefficient. Receiver operating characteristic (ROC) analysis was performed in the epithelioid subtype from the full cohort with a follow-up of $>12$ months $(n=221)$ to investigate the sensitivity and specificity of Ki67 in predicting short-term survival using the median OS of 12 months as cutoff. Furthermore, we validated three other recently published blood prognostic MPM biomarkers in patients where this information was available. About 118 patients were analysed for the neutrophil-to-lymphocyte ratio (NLR) (Kao et al, 2010; Kao et al, 2011), 97 patients for C-reactive protein (CRP) (Ghanim et al, 2012) and finally 127 patients for fibrinogen levels (Ghanim et al, 2014) using cutoffs from the aforementioned studies. In addition, in 65 patients, all three aforementioned biomarkers were available and could therefore be directly compared with each other and to Ki67 in ROC analyses. All statistical analyses were calculated with the PASW Statistics 18.0 package (Predictive Analytics Software, SPSS Inc., Chicago, IL, USA). $P$-values are given as two-sided and were considered statistically significant below 0.05 .

\section{RESULTS}

Histological subtype and treatment modality influence OS in MPM. The test cohort consisted of 40 female (21.4\%) and 147 male $(78.6 \%)$ histologically verified MPM patients $(n=187$, mean age: $60.2 \pm 10.6$ years; range: $28-82$ years). About 156 patients suffered from epithelioid MPM (83.4\%) compared with 31 nonepithelioid MPM patients (16.6\%) including 22 biphasic (11.8\%) and 9 sarcomatoid (4.8\%) cases. In the test cohort, 123 patients were diagnosed at the late stage of the disease $(78.3 \%)$. With regard to therapy, 26 patients were treated by surgery alone (13.9\%), 45 patients received multimodality therapy regimens including macroscopic radical tumour surgery $(24.1 \%), 53$ patients were treated by chemo and/or radiotherapy (28.3\%) and further 33 patients had best supportive care only (17.6\%). In 30 patients (16\% of the test cohort), treatment information was not available. In 33 patients, induction chemotherapy was applied prior to surgery.

Median OS in the test cohort was 12.0 months (CI 9.3-14.7). In univariate survival analyses, histology (Figure 1A and Table 1) and treatment (Figure 1C, Table 1) were found to have prognostic value. Meanwhile, gender, age and disease stage (Figure 1B) had no significant impact on patient's OS in univariate survival analyses of the test cohort (Table 1).

Ki67 index is not associated with histology and stage but significantly decreased in post-chemotherapy cases. Ki67 index was determined on tumour sections following immunohistochemical staining (Figure 2A). In the HUN cohort, both pathologists scored the staining to test the inter-observer reproducibility. The scoring of this cohort, by the two pathologists (US and IS), showed a strong and significant correlation (Pearson's correlation coefficient: $0.668, P<0.001, n=41$ ).

The median percentage of Ki67-positive tumour cells was $15.0 \%$ (range: $0-60 \%)$ for the entire test cohort $(n=187)$. Patient characteristics according to low and high Ki67 index dichotomized by the median are shown in Table 2 . There was no significant difference in the distribution of Ki67 index with regard to age, sex, histology or stage. The Ki67 labelling showed no association with histology (mean \pm s.d.: epithelioid $16.3 \pm 12.9$ vs non-epithelioid 18.2 $\pm 14.6, \quad P=0.465$ or epithelioid $16.3 \pm 12.9$ vs biphasic $18.3 \pm 15.3$ vs sarcomatoid 18.2 $\pm 13.6, P=0.766$, Figure $2 \mathrm{~B})$. In line with this finding, the distribution of the histological subtypes within the high and low Ki67 index groups did not differ significantly $(P=0.554$, Table 2 ; also compare representative pictures of the histological subtypes with high and low Ki67 labelling in Figure 2A). Furthermore, no association of Ki67 index and disease stage was found in the test cohort, and there was only a non-significant modest tendency towards higher Ki67 index in late stage of the disease $(17.6 \pm 13.2 v s$ early stage $13.9 \pm 13.6$, $P=0.144$, Figure 2C). However, patients in the multimodality treatment group more frequently had low Ki67 index when compared with the other treatment groups. Accordingly, the Ki67 index differed significantly between patients treated by induction therapy ( $n=33$, mean Ki67 index: $10.5 \pm 8.5)$ and the rest of the test cohort who had not received chemotherapy before sample collection $(n=124$, mean Ki67 index: $18.3 \pm 13.9, P<0.001$, Figure 2D). 

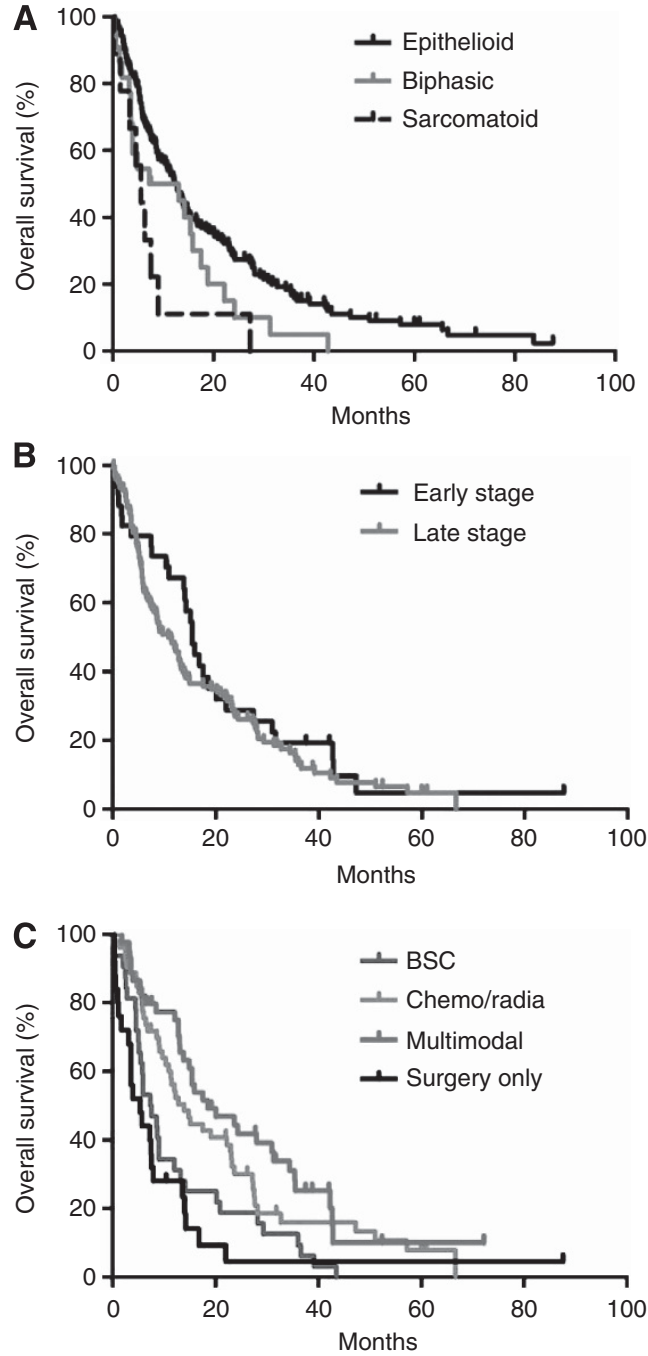

Figure 1. Overall survival prognosticators in MPM. (A) The three histological subtypes are characterised by different outcomes (epithelioid 12.8 (Cl 10.5-15.0) months vs biphasic 7.2 (Cl 0-20.7) vs sarcomatoid $5.6(\mathrm{Cl} 2.5-8.7)$ months, $P=0.005)$. (B) Disease stage had no significant impact on OS (late stage 10.8 (Cl 7.3-14.3) months vs early stage $15.4(\mathrm{Cl} 13.1-17.8)$ months, $P=0.305)$. (C) Treatment has robust prognostic impact on OS (multimodality therapy $18.5(\mathrm{Cl} 8.2-$ 28.8) months vs chemo and/or radiotherapy 13.9 (Cl 9.6-18.3) months vs best supportive care (BSC) $7.6(\mathrm{Cl} 4.4-10.8)$ months vs surgery alone 5.3 (Cl 0.8-9.7) months, $P<0.001)$.

Ki67 is an independent prognostic marker only in epithelioid MPM. MPM patients with high Ki67 index had significantly worse outcome when compared with the low Ki67 index group (HR 2.3, CI 1.7-3.2, $P<0.001)$ in univariate survival analyses of the test cohort. Accordingly, median OS in the low Ki67 group (19.1 (CI 11.7-26.5) months) was significantly $(P<0.001)$ higher than in the patients with high Ki67 index (7.5 (CI 5.2-9.8) months, Figure $3 \mathrm{~A})$. Furthermore, we performed multivariate analyses to clarify whether Ki67 is also an independent prognostic marker in MPM. We therefore utilised a multimodality Cox-regression model adjusted for age (dichotomized by the median age of 61.5 years), gender, stage (early vs late stage), histology (epithelioid $v s$ nonepithelioid MPM), treatment (surgery alone $v s$ best supportive care $v s$ chemo and or radiotherapy $v s$ multimodality therapy including macroscopic complete surgery) and Ki67 index (dichotomized by $15 \%$ ) including 149 patients with all aforementioned parameters available in the medical records (missing cases: $n=38$ ).
Table 1. Univariate survival analyses in the test cohort $(n=187)$

\begin{tabular}{|c|c|c|c|}
\hline Characteristics & OS (Cl) & $P^{a}$ & $\mathrm{HR}(\mathrm{Cl})$ \\
\hline \multicolumn{4}{|l|}{ Age, years } \\
\hline$\leqslant 61.5$ & $12.2(10.0-14.5)$ & 84 & $0.97(0.72-1.31)$ \\
\hline$>61.5$ & $10.3(5.2-15.4)$ & - & - \\
\hline \multicolumn{4}{|l|}{ Sex } \\
\hline Female & $11.3(9.6-13.0)$ & 0.17 & $0.76(0.51-1.13)$ \\
\hline Male & $12.1(8.6-15.6)$ & - & - \\
\hline \multicolumn{4}{|l|}{ Histology } \\
\hline Epithelioid & $12.8(10.5-15.0)$ & $<0.01$ & 1 \\
\hline Biphasic & $7.2(0-20.7)$ & - & - \\
\hline Sarcomatoid & $5.6(2.5-8.7)$ & - & - \\
\hline \multicolumn{4}{|l|}{ Stage $^{b}$} \\
\hline Early stage & $15.4(13.1-17.8)$ & 0.31 & $1.24(0.82-1.87)$ \\
\hline Late stage & $10.8(7.3-14.3)$ & - & - \\
\hline \multicolumn{4}{|l|}{ Treatment $^{b}$} \\
\hline Surgery alone & $5.3(0.8-9.7)$ & $<0.01$ & $1.30(0.76-2.21)$ \\
\hline Best supportive care & $7.6(4.4-10.8)$ & - & 1 \\
\hline Chemo and/or radiotherapy & $13.9(9.6-18.3)$ & - & $0.61(0.39-0.95)$ \\
\hline Multimodality treatment & $18.5(8.2-28.8)$ & - & $0.46(0.28-0.74)$ \\
\hline
\end{tabular}

After multivariate survival analyses, Ki67 index proved to be an independent prognostic marker in MPM (HR: 2.1, CI: 1.4-3.1, $P<0.001)$ beside histology $(P=0.013)$ and treatment modality $(P=0.001)$, whereas age, gender and stage had no independent prognostic impact on OS of the test cohort as shown in Table 3. After subgroup analyses, Ki67 only showed a prognostic power in the epithelioid subtype (Ki67 low: 22.9 (CI 17.8-27.9) months vs Ki67 high: 7.9 (CI 5.3-10.4) months, $P<0.001$ Figure 3B) whereas it had no prognostic impact in the non-epithelioid subtype (Ki67 low: 4.9 (CI 0-10.9) vs Ki67 high: 6.3 (CI 2.4-10.2) months, $P=0.630)$ as shown in Figure 3C. In all other subgroup analyses (for age, gender, stage and treatment), Ki67 proved to beaccording to the multivariate analysis-a prognostic marker in all except the surgery-alone subgroup. Accordingly, an additional analysis was performed to investigate whether surgical treatment influences the prognostic power of Ki67. Interestingly, Ki67 proved to be a prognosticator both for surgically treated patients (multimodality group and surgery-alone group high vs low Ki67 OS: 7.9 vs 18.5 months; $P=0.001$ ) as well as for patients without surgical treatment regimens (chemo and/or radiotherapy alone and best supportive care group high vs low Ki67 OS: 7.6 vs 22.9 months; $P<0.001)$.

Ki67 index is a reproducible prognostic marker. To confirm the prognostic value of Ki67 in MPM, we have analysed the independent SLO cohort $(n=98 ; 21$ female, 77 male; mean age: $66.9 \pm 9.0$ years; range: $42-84$ years). Median Ki67 count in the SLO validation cohort was $22 \%$ (range: 2-70.) Accordingly, we dichotomized this cohort also by its median Ki67 index, resulting in 46 patients allocated to the high $(>22 \%)$ and 52 patients allocated to the low Ki67 group. Again, patients with high Ki67 index had significantly worse outcome when compared with those with low Ki67 index. OS was 18.2 months in the low Ki67 group (CI 12.4-24.1 months) vs 9.1 months (CI 5.4-12.9 months, log rank test: $P=0.048$; Figure $3 \mathrm{D}$ ) in the high Ki67 expression group.

Ki67 is an accurate marker predicting short-term survival in epithelioid MPM. To test the sensitivity and specificity for detecting early death (death before median OS of 12 months was reached), ROC curve analysis was performed. We therefore 
A
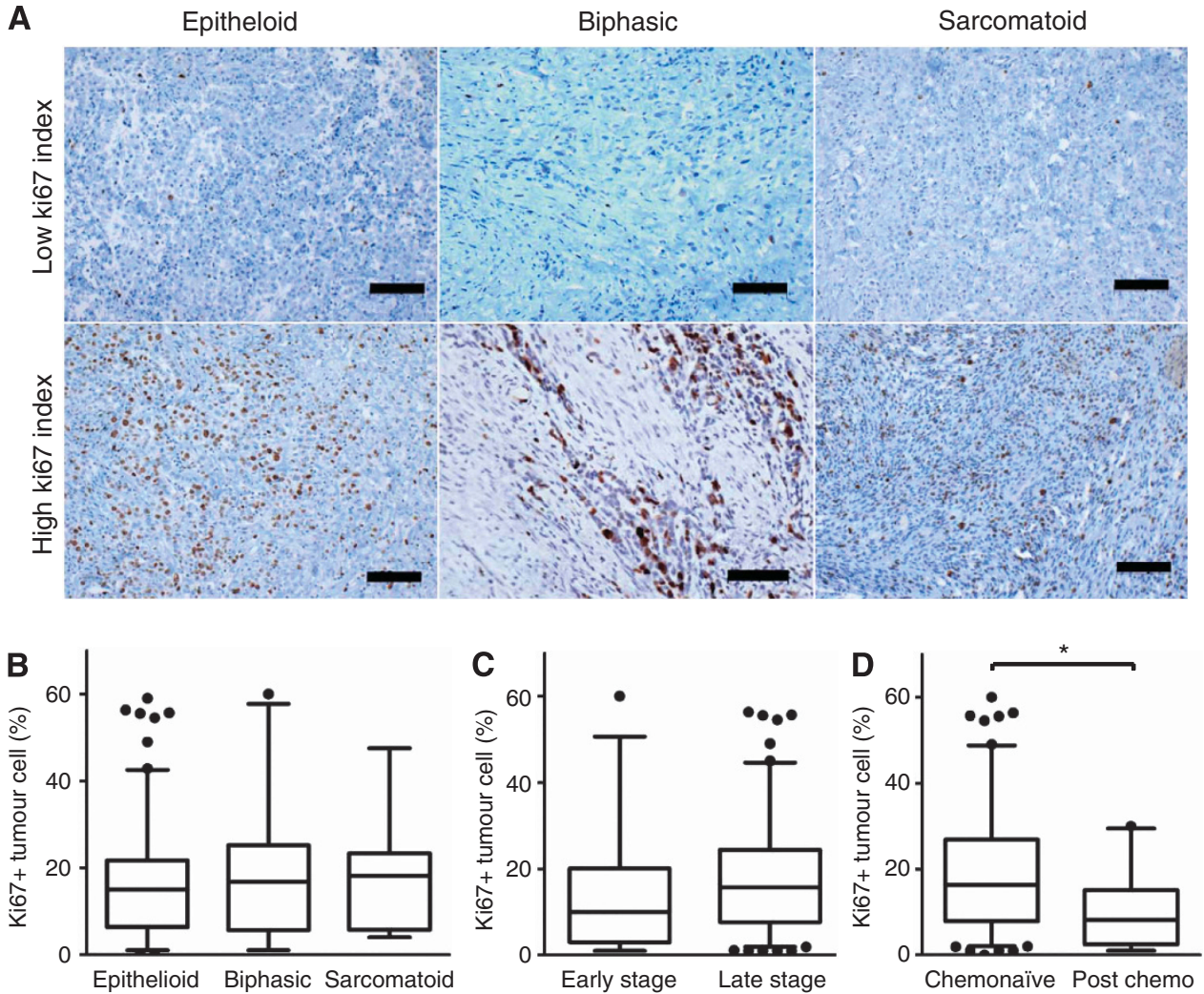

Figure 2. Ki67 index in MPM tissue samples. (A) Ki67 nuclear staining in all three major histological subtypes of MPM. Scale bar is $100 \mu \mathrm{m}$. (B) There was no significant difference between the three histological subtypes with regard to Ki67 index (mean \pm s.d.: epithelioid $16.3 \pm 12.9$ vs biphasic $18.3 \pm 15.3$ vs sarcomatoid 18.2 $\pm 13.6, P=0.766$ ). (C) Modest increase of Ki67 index in late stage of disease (mean \pm s.d.: $17.6 \pm 13.2)$ vs early stage $(13.9 \pm 13.6, P=0.144)$. (D) Patients after induction chemotherapy had significantly lower amount of Ki67-positive tumour cells when compared with the treatment naïve patients in the test cohort (mean \pm s.d.: induction chemotherapy $10.5 \pm 8.5$ vs chemo-naïve 18.3 \pm 13.9 , asterisk denotes significance of $P<0.001$ ).

analysed all epithelioid MPM patients with clinical follow-up for at least 12 months $(n=221)$. In this analysis, Ki67 showed an area under the curve of 0.68 (Figure 4A). The sensitivity and specificity at $15 \%$ (the cutoff value used in the test cohort) were 0.69 and 0.57 , respectively.

Comparison of Ki67 index and prognostic blood parameters. A number of inflammation- related blood parameters have recently been reported to reflect the prognosis of MPM. Therefore the correlation of Ki67 index to NLR, fibrinogen and CRP has been studied. Ki67 only showed a weak albeit significant correlation to fibrinogen ( $n=127$, Pearson's correlation coefficient 0.324 , $P<0.001$ ) but not to the other proinflammatory markers (CRP: $n=97$ and NLR: $n=118$ ). Of note, a significant correlation $(n=92$, Pearson's correlation coefficient $0.530, P<0.001)$ was found between the two acute phase proteins, namely fibrinogen and CRP.

Nevertheless, patients with high Ki67 index had more frequently elevated CRP $(P=0.001, n=97)$, fibrinogen $>627 \mathrm{mg} \mathrm{dl}^{-1}$ $(P<0.001, n=127)$ and a (non-significant) tendency to have a NLR $>5(P=0.160, n=118)$. Next, we performed subgroup analyses to clarify if Ki67 index adds prognostic information on top of the prognostic power provided by the proinflammatory parameters. Interestingly, Ki67 was found to be prognostic in patients with elevated CRP $(P=0.022)$. Due to the low number $(n=3)$ of patients with normal CRP but high Ki67 index, the normal CRP subgroup could not be analysed. With regard to fibrinogen, Ki67 was prognostic in both the high $(P=0.028)$ and low $(P=0.005)$ fibrinogen groups. Furthermore, Ki67 was also found to be prognostic in both the high $(P=0.001)$ and low
$(P<0.001)$ NLR groups, indicating that indeed Ki67 adds prognostic information to the recently published proinflammatory parameters.

To compare the prognostic power of these three aforementioned MPM biomarkers with Ki67, an additional ROC analysis was performed. Ki67, NLR, fibrinogen and CRP showed areas under the curve of $0.70,0.72,0.62$ and 0.71 , respectively, indicating that Ki67 index carries comparable prognostic power to these recently published prognostic proinflammatory markers (Figure 4B).

Comparison of the nuclear grading score with Ki67 index and other prognostic parameters. Finally, we wanted to compare the prognostic value of Ki67 index and the nuclear grading score as previously described by Kadota et al (2012). To compare all prognostic parameters, we determined the nuclear grading score for the patients included in the ROC analysis. The nuclear grading score was established for 59 patients. With regard to nuclear atypia, 3 patients were allocated to the mild and 33 patients to the moderate nuclear atypia group. Accordingly, we compared these 36 patients to 23 patients with tumours characterised by severe atypia. Based on mitotic count analysis, 20 patients were allocated to the low, 20 patients to the intermediate and 19 patients to the high mitosis group. In this case, we combined low and intermediate groups and contrasted them to the high mitotic count patients. Altogether, 35 and 24 patients had low (2-4) and high (5 and 6) nuclear grading score, respectively.

Patients with mild or moderate nuclear atypia had significant longer median OS (14.1, CI 8.2-20.1 months) than patients with severe nuclear atypia (9.1, CI 2.8-15.4 months, $P=0.022$ ). Furthermore, Ki67 expression was significantly higher in the 
Table 2. Patient characteristics and distribution according to Ki67 expression in the test cohort $(n=187)$

\begin{tabular}{|c|c|c|c|c|c|c|c|}
\hline \multirow[b]{2}{*}{ Characteristics } & \multicolumn{2}{|c|}{ Low Ki67 } & \multicolumn{2}{|c|}{ High Ki67 } & \multicolumn{2}{|c|}{ All patients } & \multirow[b]{2}{*}{$P$-value } \\
\hline & Numbers & $\%$ & Numbers & $\%$ & Numbers & $\%$ & \\
\hline \multicolumn{8}{|l|}{ Age, Years } \\
\hline $\begin{array}{l}\leqslant 61.5 \\
>61.5\end{array}$ & $\begin{array}{l}50 \\
45\end{array}$ & $\begin{array}{l}53.2 \\
48.4\end{array}$ & $\begin{array}{l}44 \\
48\end{array}$ & $\begin{array}{l}46.8 \\
51.6\end{array}$ & $\begin{array}{l}94 \\
93\end{array}$ & $\begin{array}{l}50.3 \\
49.7\end{array}$ & $\begin{array}{l}\mathrm{NS}^{*} \\
-\end{array}$ \\
\hline \multicolumn{8}{|l|}{ Sex } \\
\hline $\begin{array}{l}\text { Female } \\
\text { Male }\end{array}$ & $\begin{array}{l}25 \\
70\end{array}$ & $\begin{array}{l}62.5 \\
47.6\end{array}$ & $\begin{array}{l}15 \\
77\end{array}$ & $\begin{array}{l}37.5 \\
52.4\end{array}$ & $\begin{array}{r}40 \\
147\end{array}$ & $\begin{array}{l}21.4 \\
78.6\end{array}$ & $\mathrm{NS}^{*}$ \\
\hline \multicolumn{8}{|l|}{ Histology } \\
\hline $\begin{array}{l}\text { Epithelioid } \\
\text { Non- Epithelioid }\end{array}$ & $\begin{array}{l}81 \\
14\end{array}$ & $\begin{array}{l}51.9 \\
45.2\end{array}$ & $\begin{array}{l}75 \\
17\end{array}$ & $\begin{array}{l}48.1 \\
54.8\end{array}$ & $\begin{array}{r}156 \\
31\end{array}$ & $\begin{array}{l}83.4 \\
16.6\end{array}$ & $\begin{array}{l}\mathrm{NS}^{*} \\
-\end{array}$ \\
\hline \multicolumn{8}{|l|}{ Stage\# } \\
\hline $\begin{array}{l}\text { early stage } \\
\text { late stage }\end{array}$ & $\begin{array}{l}22 \\
60\end{array}$ & $\begin{array}{l}64.7 \\
48.8\end{array}$ & $\begin{array}{l}12 \\
63\end{array}$ & $\begin{array}{l}35.3 \\
51.2\end{array}$ & $\begin{array}{r}34 \\
123\end{array}$ & $\begin{array}{l}21.7 \\
78.3\end{array}$ & $\mathrm{NS}^{*}$ \\
\hline \multicolumn{8}{|l|}{ Treatment\# } \\
\hline $\begin{array}{l}\text { Surgery alone } \\
\text { Best supportive care } \\
\text { Chemo and/or Radiotherapy } \\
\text { Multimodal treatment }\end{array}$ & $\begin{array}{l}12 \\
13 \\
24 \\
32\end{array}$ & $\begin{array}{l}46.2 \\
39.4 \\
45.3 \\
71.1\end{array}$ & $\begin{array}{l}14 \\
20 \\
29 \\
13\end{array}$ & $\begin{array}{l}53.8 \\
60.6 \\
54.7 \\
28.9\end{array}$ & $\begin{array}{l}26 \\
33 \\
53 \\
45\end{array}$ & $\begin{array}{l}16.6 \\
21 \\
33.8 \\
28.7\end{array}$ & $\begin{array}{c}0.02^{\star} \\
- \\
- \\
-\end{array}$ \\
\hline \multicolumn{8}{|l|}{ Surgery\# } \\
\hline $\begin{array}{l}\text { Surgery } \\
\text { No Surgery }\end{array}$ & $\begin{array}{l}44 \\
37\end{array}$ & $\begin{array}{l}62 \\
43\end{array}$ & $\begin{array}{l}27 \\
49\end{array}$ & $\begin{array}{l}38 \\
57\end{array}$ & $\begin{array}{l}71 \\
86\end{array}$ & $\begin{array}{l}45.2 \\
54.8\end{array}$ & $\begin{array}{c}0.02^{\star} \\
-\end{array}$ \\
\hline \multicolumn{8}{|l|}{ Induction Therapy\# } \\
\hline $\begin{array}{l}\text { Induction } \\
\text { Chemo-naïve at sampling } \\
\text { time }\end{array}$ & $\begin{array}{l}25 \\
56\end{array}$ & $\begin{array}{l}75.8 \\
45.2\end{array}$ & $\begin{array}{r}8 \\
68\end{array}$ & $\begin{array}{l}24.2 \\
54.8\end{array}$ & $\begin{array}{r}33 \\
124\end{array}$ & $\begin{array}{l}21 \\
79\end{array}$ & $\begin{array}{c}0.01^{\star} \\
-\end{array}$ \\
\hline
\end{tabular}
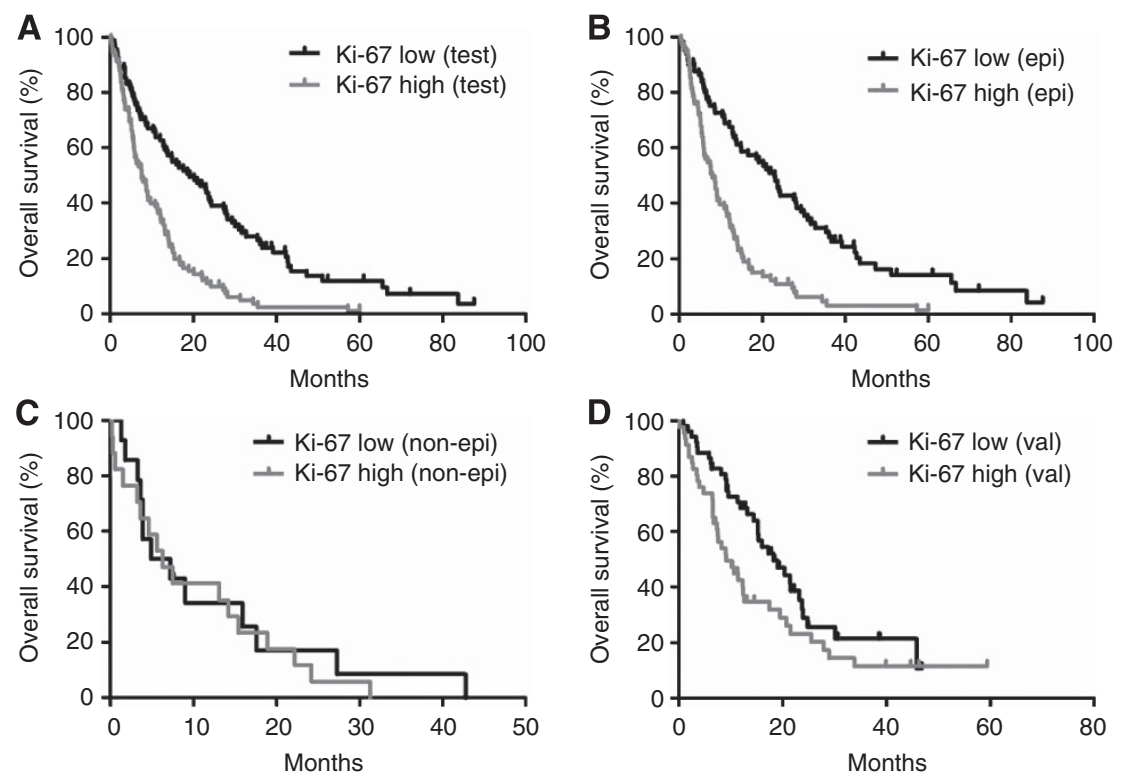

Figure 3. Prognostic power of Ki67 index in MPM. (A) Kaplan-Meier survival analysis of the test cohort $(n=187)$. There was a significant difference in the OS between patients with high $(n=92)$ vs low $(n=95) \mathrm{Ki67}$ index (HR 2.3, Cl 1.7-3.2, $P<0.001)$. Median OS was higher in low Ki67 index $(<15 \%)$ group (19.1 (Cl 11.7-26.5) months) than in patients with high Ki67 index (7.5 (Cl 5.2-9.8) months, $P<0.001))$. (B) Kaplan-Meier survival curve shows that Ki67 is prognostic in epithelioid MPM. (C) In contrast, there is no impact of Ki67 index on OS in patients with non-epithelioid MPM $(n=31)$. (D). Kaplan-Meier survival analysis in the validation cohort $(n=98)$. Ki67 had a significant impact on OS $(P=0.048)$ when using median Ki67 expression of the validation cohort as cutoff (22\%).

severe nuclear atypia group when compared with the mild and moderate atypia groups (mean \pm s.d.: $25.43 \pm 16.22$ vs $15.58 \pm 11.06, P=0.015)$.

Patients with high mitotic count had a-not significanttendency for shorter median OS (11.3, CI 2.3-20.3 months) when compared with those with intermediate and low mitotic counts (13.6, CI 11.9-15.2 months, $P=0.063$ ). As expected, the Ki67 index was significantly higher in the high mitotic count group when compared with the low and intermediate count group (mean \pm s.d.: $27.3 \pm 13.9$ vs $15.7 \pm 12.6, P=0.002$ ). 
With regard to nuclear grading score, the low score group had significantly longer OS (14.9, CI 8.5-21.3 months) when compared with the high score group (9.1, CI 2.4-15.9, $P=0.019)$. According to the previous analyses, Ki67 index was significantly higher in the high nuclear grading group when compared with the low nuclear grading group (mean \pm s.d.: $27.6 \pm 14.1$ vs $13.8 \pm 11.1, P<0.001$ ).

The inflammatory related marker CRP tended to be higher in the high nuclear grading group $\left(5.78 \pm 3.98 \mathrm{mg} \mathrm{dl}^{-1}\right)$ when compared with the low nuclear grading group $\left(4.14 \pm 4.26 \mathrm{mg} \mathrm{dl}^{-1}\right.$, $P=0.140$ ). A similar tendency was observed with regard to mean fibrinogen levels in the nuclear grading groups high $v s$ low $\left(675.71 \pm 225.13\right.$ vs $572.49 \pm 188.90 \mathrm{mg} \mathrm{dl}^{-1}$, respectively, $P=0.062$ ). Also for the NLR, the nuclear grading tended to be more frequently high in the high fibrinogen group when compared with the low fibrinogen group (Pearsons $\chi^{2}$-test, $P=0.166$ ). Finally, we performed a univariate analysis for predicting an OS

\section{Table 3. Cox-regression model adjusted for patient}

characteristics of all test cohort patients with available complete data for model adjustment $(n=149)$

\begin{tabular}{|c|c|c|c|}
\hline Characteristics & $\begin{array}{c}\text { Adjusted HR for } \\
\text { death }\end{array}$ & $95 \% \mathrm{Cl}$ & $\begin{array}{c}\text { Adjusted } \\
P \text {-value }\end{array}$ \\
\hline \multicolumn{4}{|l|}{ Age, years } \\
\hline $\begin{array}{l}\leqslant 61.5 \\
>61.5\end{array}$ & $\begin{array}{l}0.98 \\
1\end{array}$ & $\begin{array}{c}0.69-1.40 \\
-\end{array}$ & $\begin{array}{l}0.92 \\
-\end{array}$ \\
\hline \multicolumn{4}{|l|}{ Sex } \\
\hline $\begin{array}{l}\text { Female } \\
\text { Male }\end{array}$ & $\begin{array}{l}0.94 \\
1\end{array}$ & $\begin{array}{c}0.53-1.67 \\
-\end{array}$ & $\begin{array}{l}0.84 \\
-\end{array}$ \\
\hline \multicolumn{4}{|l|}{ Histology } \\
\hline $\begin{array}{l}\text { Epithelioid } \\
\text { Non-epithelioid }\end{array}$ & $\begin{array}{l}1 \\
1.90\end{array}$ & $\overline{1.14-3.15}$ & $\begin{array}{l}0.01 \\
-\end{array}$ \\
\hline \multicolumn{4}{|l|}{ Stage } \\
\hline $\begin{array}{l}\text { Early stage } \\
\text { Late stage }\end{array}$ & $\begin{array}{l}0.64 \\
1\end{array}$ & $\begin{array}{c}0.36-1.12 \\
-\end{array}$ & $\begin{array}{l}0.12 \\
-\end{array}$ \\
\hline \multicolumn{4}{|l|}{ Treatment } \\
\hline $\begin{array}{l}\text { Surgery alone } \\
\text { Best supportive care } \\
\text { Chemo and/or } \\
\text { Radiotherapy } \\
\text { Multimodal treatment }\end{array}$ & $\begin{array}{l}1.44 \\
1 \\
0.58 \\
0.52\end{array}$ & $\begin{array}{c}0.75-2.80 \\
- \\
0.36-0.93 \\
0.30-0.91\end{array}$ & $\begin{array}{l}0.28 \\
- \\
0.02 \\
0.02\end{array}$ \\
\hline \multicolumn{4}{|l|}{ Ki67 Expression } \\
\hline $\begin{array}{l}\text { Low Ki67 } \\
\text { High Ki67 }\end{array}$ & $\begin{array}{l}1 \\
2.11\end{array}$ & $\frac{-}{1.44-3.10}$ & $<0.01$ \\
\hline
\end{tabular}

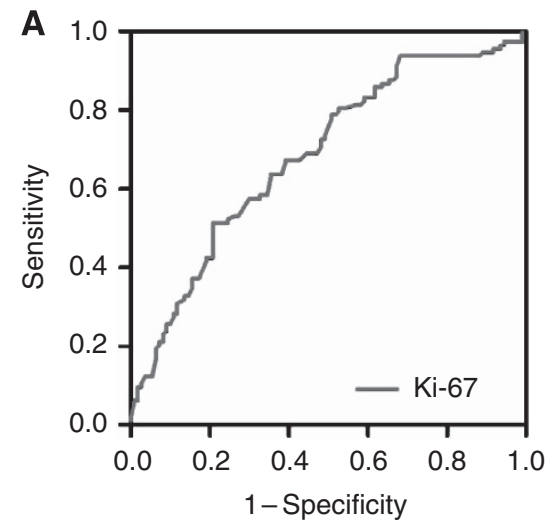

of $<12$ months for all biomarkers studied (Table 4). All factors, but the mitotic count, were significant prognosticators.

\section{DISCUSSION}

In the present study, we performed a comprehensive analysis of the prognostic power of Ki67 index in MPM. Besides known prognostic factors such as histological subtype and treatment modality, Ki67 index was found to be an OS prognosticator in a test cohort of 187 patients. The large international patient cohort provided us the opportunity to show that the prognostic value of the proliferation index is indeed independent of age, sex, stage, histology and treatment in the test cohort patients with full clinical record $(n=149)$. In addition, we were able to reproduce and verify the prognostic power of this routine proliferation marker in an independent cohort of 98 patients from a different centre. Furthermore, we were able to compare the prognostic power of Ki67 index with other recently published prognostic markers including CRP (Ghanim et al, 2012), fibrinogen (Ghanim et al, 2014), NLR (Kao et al, 2010; Kao et al, 2011) and nuclear grading score (Kadota et al, 2012).

In MPM, Ki67 expression detected in serous effusions was described to correlate with OS, but did not reach statistical significance in a series of 20 patients (Schonherr et al, 2004). Furthermore, Ki67 index was found to differ significantly between long- and short-term MPM survivors in three small series in the past (Comin et al, 2000; Bongiovanni et al, 2001; Leonardo et al, 2001). In a more recent study, a nuclear grading system-that includes mitotic index-was found to be prognostic in the epithelioid subtype of MPM (Kadota et al, 2012) in tissue microarray analyses when using $10 \%$ as cutoff. In addition, Bitanihirwe et al (2014), found a prognostic value of Ki67 in treatment-naïve MPM patients as well as in patients after induction therapy in tissue microarray analyses. In the aforementioned study, a cutoff of $20 \%$ Ki67-positive tumour cells was used in chemonaïve patients, whereas $11.25 \%$ was used as cutoff in patients after induction therapy (Bitanihirwe et al, 2014). Importantly, our study on whole FFPE blocks-a clinically more relevant material as compared with TMA - used similar cutoff values for the respective subgroups. Of note, when using the cutoff value for induction therapy patients from the study of Bitanihirwe et al (2014), $(11.25 \%)$, Ki67 index was prognostic in the induction therapy subcohort from our study (OS: 30.9 vs 15.4 months, $P=0.020$, $n=33)$.

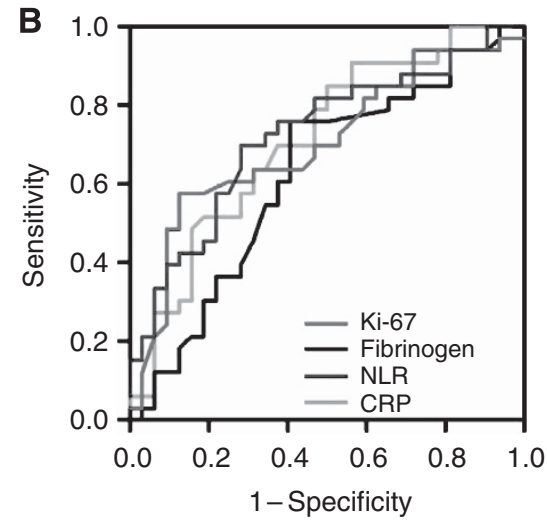

Figure 4. Sensitivity and specificity analyses of Ki67 by ROC analysis. (A) in all epithelioid MPM patients with follow-up of at least 12.0 months $(n=221)$ showed an area under the curve of 0.68 . The sensitivity and specificity at $15 \%$ (cutoff used in the test cohort) were 0.69 and 0.57 , respectively. (B) Ki67 index, neutrophil-to-lymphocyte ratio (NLR), fibrinogen and C-reactive protein (CRP) showed areas under the curve of 0.70 , $0.72,0.62$ and 0.71 , respectively $(n=65)$. 
Table 4. Comparison of prognostic biomarkers in univariate survival analyses

\begin{tabular}{|l|c|c|c|}
\hline $\begin{array}{l}\text { Characteristics } \\
\text { C-reactive protein }\end{array}$ & $\begin{array}{c}\text { Number of } \\
\text { patients }\end{array}$ & $P^{\mathrm{a}}$ & $\mathrm{HR}(\mathrm{Cl})$ \\
\hline $\begin{array}{l}\leqslant 1 \mathrm{mg} \mathrm{dl}^{-1} \\
>1 \mathrm{mg} \mathrm{dl}^{-1}\end{array}$ & -97 & $<0.01$ & $2.55(1.39-4.65)$ \\
- & - & - \\
\hline Fibrinogen $^{\leqslant}$ & - & $<0.01$ & $1.85(1.26-2.72)$ \\
$>627 \mathrm{mg} \mathrm{dl}^{-1}$ & -127 & - & - \\
\hline NLR dl & -1 \\
\hline $\begin{array}{l}<5 \\
\geqslant 5\end{array}$ & - & -0.01 & $1.76(1.14-2.71)$ \\
\hline
\end{tabular}

\section{Nuclear atypia}

\begin{tabular}{|l|c|c|c|}
\hline Mild and moderate & 59 & 0.03 & $1.97(1.09-3.57)$ \\
Severe & - & - & - \\
\hline
\end{tabular}

\begin{tabular}{|l|c|c|c|}
\hline Mitotic count \\
$\begin{array}{l}\text { Low and } \\
\text { intermediate } \\
\text { High }\end{array}$ & 59 & 0.07 & $0.58(0.33-1.04)$ \\
\hline Nuclear grading score & - & - \\
\hline $\begin{array}{l}\text { Low (2-4) } \\
\text { High (5 and 6) }\end{array}$ & - & -0.02 & $1.95(1.11-3.45)$ \\
\hline Ki67 index & - & - & - \\
\hline $\begin{array}{l}\leqslant 15 \\
>15\end{array}$ & 59 & $<0.01$ & $2.58(1.42-4.69)$ \\
\hline
\end{tabular}

Abbreviations: $\mathrm{Cl}=$ confidence interval; $\mathrm{HR}=$ hazard ratio; NLR-neutrophil-to-lymphocyte ratio.

${ }^{\mathrm{a}}$ Cox regression

Importantly, we hereby describe for the first time that Ki67 is only prognostic in the epithelioid but not in the non-epithelioid histological subtype of MPM. Similar to Hirano et al (2002), we could not show a significant difference in the proportion of Ki67positive tumour cells between the epithelioid and non-epithelioid histological subtypes (Figures 2A and B). Previously, Kadota et al (2012) only analysed epithelioid MPM patients and the very recent study of Bitanihirwe et al (2014) did not report subgroup analyses with regard to Ki67 and histology. However, when using the cutoff from the study by Kadota et al (2012) (10\%), Ki67 again was only prognostic in the epithelioid $(P<0.001)$ but not in the nonepithelioid subtype $(P=0.715)$.

Furthermore, in our study, Ki67 index was also not associated with other clinical characteristics, such as gender, age or tumour stage but showed a-not significant-tendency to be more frequently increased in late stage of disease as also shown in Figure 2C. Of note, high Ki67 index was also associated with the proinflammatory phenotype-reflected by elevation of the recently published MPM prognosticators CRP (Ghanim et al, 2012), fibrinogen (Ghanim et al, 2014) and the NLR (Kao et al, 2010; Kao et al, 2011)-indicating that an elevated systemic immune response of the patient is associated with a more proliferative tumour phenotype. In line with these findings, the nuclear grading score tended to be associated with a proinflammatory status. Nevertheless, our study demonstrated that ki67 index was prognostic in both subgroups of patients dichotomized by the inflammation-related parameters. These observations argues for a more aggressive biological MPM subtype associated with high Ki67 expression and might, in addition to tumour stage and proinflammatory phenotype, help pathologists to further classify MPM according to their biological behaviour. However, in subgroup analyses, Ki67 was prognostic in both early as well as late stage of disease excluding the fact that Ki67 expression simply reflects clinically undetected late stage of disease.
Importantly, we could detect significant differences of Ki67 expression between the treatment groups. This observation can be explained by the high number of patients after induction therapy in the multimodality therapy group (33 out of 45 patients in the multimodality group received induction chemotherapy before surgery/sampling of the tumour). Figure 2D shows the lower amount of Ki67-positive tumour cells in patients after induction chemotherapy, reflecting the lower number of mitotic tumour cells after chemotherapy. This is in line with the recently published observations of Bitanihirwe et al (2014) and, therefore, suggests that Ki67 index might also be a clinically useful marker to estimate biological response achieved by (induction-) chemotherapy. Again, the treatment subgroup analyses demonstrated that Ki67 was prognostic in all treatment cohorts except the surgery-alone group. However, the surgery-alone group was the smallest and resulted in the worst outcome. This may impair the proper statistical analysis of this subgroup in terms of prognostic power. Furthermore, we showed that Ki67 was prognostic in both surgically treated patients (multimodality therapy group and surgery-alone group) as well as in non-surgically treated patients (chemo and/or radiotherapy alone and best supportive care patients). Multivariate survival analyses proved that the prognostic value of Ki67 is independent of age, sex, stage, histology and treatment modality. Besides Ki67 expression, univariate survival analyses revealed-in line with previous studies (Aigner et al, 2008; van Zandwijk et al, 2013)_ histology and treatment as prognostic factors. Also, the recently published proinflammatory biomarkers CRP (Ghanim et al, 2012), fibrinogen (Ghanim et al, 2014) and NLR (Kao et al, 2010; Kao et al, 2011) were found to have prognostic value in this large MPM cohort. Furthermore, we could also validate the prognostic power of the nuclear grading score (Kadota et al, 2012). After multivariate analyses, Ki67 index, histological subtype and treatment remained as significant prognosticators. Patients who received surgery as single treatment modality survived significantly shorter than those treated within multimodality treatment protocols as previously published by our group (Aigner et al, 2008). Our multicentre study with a large patient population strongly supports the importance of interdisciplinary treatment in MPM.

We also demonstrated that Ki67 index has a prognostic power similar to other recently described inflammation-related blood parameters (Kao et al, 2010; Kao et al, 2011; Ghanim et al, 2012; Ghanim et al, 2014). In the ROC analysis, Ki67 index was found to be an accurate prognosticator with an area under the curve of 0.70 . Importantly, there was a strong correlation between fibrinogen and CRP, suggesting that these two acute phase proteins reflect the same biological characteristics of MPM patients. At the same time, NLR and Ki67 index did not correlate, indicating that these two parameters might deliver additional biological information in MPM patients. Of note, in our study cohort, CRP, fibrinogen and NLR were also found to be of prognostic value when all three markers were analysed as metric characteristics. Our finding thus supports the prognostic power of NLR that is recently heavily debated (Meniawy et al, 2013; Kao et al, 2014; Meniawy et al, 2014).

In summary, the current study demonstrates that Ki67 index is an accurate prognostic marker in epithelioid MPM indicating the proportion of proliferating tumour cells and reflecting a more aggressive biological behaviour. It can significantly contribute to the estimation of the course of the disease as other studies-similar to our findings-have indicated that the current staging system does not have sufficient prognostic power (Edwards et al, 2000; Tanrikulu et al, 2010). Accordingly, the histological classification and staging of MPM needs further improvement to better reflect biological behaviour and consequently estimate more precisely the clinical outcome of MPM patients.

In the present study, Ki67 index demonstrated significant prognostic power in two independent study cohorts. The introduction 
of Ki67 index as a prognostic tool into the daily pathological routine work-up of MPM is feasible as Ki67 is already in use as a prognostic marker in other cancer types and, furthermore, immunohistochemical protocols for detection of Ki67 expression are relatively inexpensive, reliable and used worldwide. We, therefore, conclude that Ki67 index is useful to estimate the prognosis of epithelioid MPM and might also be a novel tool to evaluate the biological treatment response after induction chemotherapy.

\section{ACKNOWLEDGEMENTS}

We thank Sabine El Gazzar, Elisabeth Rabensteiner, Anita Brandstetter and Violetta Piurkó for their help with immunohistochemistry staining. This study was supported by KTIA AIK 12-1-2013-0041 (AR, FR-V, VL and BD); TÁMOP 424A/1-11-12012-0001 (BD); OTKA K109626, OTKA K108465 (BD and BH), OTKA MOB80325 (BH); EUREKA_HU_12-1-2012-0057 (BD); the Oesterreichische Nationalbank (Anniversary Fund), project number: 14043 (BG, AR, VL and BD) and 14574 (MAH) and the Vienna Fund for Innovative Interdisciplinary Cancer Research (BD and VL).

\section{CONFLICT OF INTEREST}

The authors declare no conflict of interest.

\section{REFERENCES}

Aigner C, Hoda MA, Lang G, Taghavi S, Marta G, Klepetko W (2008) Outcome after extrapleural pneumonectomy for malignant pleural mesothelioma. Eur J Cardiothorac Surg 34(1): 204-207.

Baratti D, Kusamura S, Cabras AD, Bertulli R, Hutanu I, Deraco M (2013) Diffuse malignant peritoneal mesothelioma: long-term survival with complete cytoreductive surgery followed by hyperthermic intraperitoneal chemotherapy (HIPEC). Eur J Cancer 49(15): 3140-3148.

Beer TW (2001) Immunohistochemical MIB-1 and p27 as prognostic factors in pleural mesothelioma. Pathol Res Pract 197(12): 859.

Bitanihirwe BK, Meerang M, Friess M, Soltermann A, Frischknecht L, Thies S, Felley-Bosco E, Tsao MS, Allo G, de Perrot M, Seifert B, Moch H, Stahel R, Weder W, Opitz I (2014) PI3K/mTOR signaling in mesothelioma patients treated with induction chemotherapy followed by extrapleural pneumonectomy. J Thorac Oncol 9(2): 239-247.

Bongiovanni M, Cassoni P, De Giuli P, Viberti L, Cappia S, Ivaldi C, Chiusa L, Bussolati G (2001) p27(kip1) immunoreactivity correlates with long-term survival in pleural malignant mesothelioma. Cancer 92(5): 1245-1250.

Comin CE, Anichini C, Boddi V, Novelli L, Dini S (2000) MIB-1 proliferation index correlates with survival in pleural malignant mesothelioma. Histopathology 36(1): 26-31.

Dingemans AM, Witlox MA, Stallaert RA, van der Valk P, Postmus PE, Giaccone G (1999) Expression of DNA topoisomerase IIalpha and topoisomerase IIbeta genes predicts survival and response to chemotherapy in patients with small cell lung cancer. Clin Cancer Res 5(8): 2048-2058.

Edwards JG, Abrams KR, Leverment JN, Spyt TJ, Waller DA, O'Byrne KJ (2000) Prognostic factors for malignant mesothelioma in 142 patients: validation of CALGB and EORTC prognostic scoring systems. Thorax 55(9): 731-735.

Gerdes J, Lemke H, Baisch H, Wacker HH, Schwab U, Stein H (1984) Cell cycle analysis of a cell proliferation-associated human nuclear antigen defined by the monoclonal antibody Ki-67. J Immunol 133(4): 1710-1715.

Ghanim B, Hoda MA, Klikovits T, Winter MP, Alimohammadi A, Grusch M, Dome B, Arns M, Schenk P, Jakopovic M, Samarzija M, Brcic L, Filipits M Laszlo V, Klepetko W, Berger W, Hegedus B (2014) Circulating fibrinogen is a prognostic and predictive biomarker in malignant pleural mesothelioma. Br J Cancer 110(4): 984-990.
Ghanim B, Hoda MA, Winter MP, Klikovits T, Alimohammadi A, Hegedus B, Dome B, Grusch M, Arns M, Schenk P, Pohl W, Zielinski C, Filipits M, Klepetko W, Berger W (2012) Pretreatment serum C-reactive protein levels predict benefit from multimodality treatment including radical surgery in malignant pleural mesothelioma: a retrospective multicenter analysis. Ann Surg 256(2): 357-362.

Hasteh F, Lin GY, Weidner N, Michael CW (2010) The use of immunohistochemistry to distinguish reactive mesothelial cells from malignant mesothelioma in cytologic effusions. Cancer Cytopathol 118(2): 90-96.

Hirano H, Tsuji M, Kizaki T, Sashikata T, Yoshi Y, Okada Y, Mori H (2002) Expression of matrix metalloproteinases, tissue inhibitors of metalloproteinase, collagens, and Ki67 antigen in pleural malignant mesothelioma: an immunohistochemical and electron microscopic study. Med Electron Microsc 35(1): 16-23.

Husain AN, Colby T, Ordonez N, Krausz T, Attanoos R, Beasley MB, Borczuk AC, Butnor K, Cagle PT, Chirieac LR, Churg A, Dacic S, Fraire A, Galateau-Salle F, Gibbs A, Gown A, Hammar S, Litzky L, Marchevsky AM, Nicholson AG, Roggli V, Travis WD, Wick M (2013) Guidelines for pathologic diagnosis of malignant mesothelioma: 2012 update of the consensus statement from the International Mesothelioma Interest Group. Arch Pathol Lab Med 137(5): 647-667.

Kadota K, Suzuki K, Colovos C, Sima CS, Rusch VW, Travis WD, Adusumilli PS (2012) A nuclear grading system is a strong predictor of survival in epitheloid diffuse malignant pleural mesothelioma. Mod Pathol 25(2): 260-271.

Kahlos K, Soini Y, Paakko P, Saily M, Linnainmaa K, Kinnula VL (2000) Proliferation, apoptosis, and manganese superoxide dismutase in malignant mesothelioma. Int J Cancer 88(1): 37-43.

Kao SC, Klebe S, Henderson DW, Reid G, Chatfield M, Armstrong NJ, Yan TD, Vardy J, Clarke S, van Zandwijk N, McCaughan B (2011) Low calretinin expression and high neutrophil-to-lymphocyte ratio are poor prognostic factors in patients with malignant mesothelioma undergoing extrapleural pneumonectomy. J Thorac Oncol 6(11): 1923-1929.

Kao SC, Pavlakis N, Harvie R, Vardy JL, Boyer MJ, van Zandwijk N, Clarke SJ (2010) High blood neutrophil-to-lymphocyte ratio is an indicator of poor prognosis in malignant mesothelioma patients undergoing systemic therapy. Clin Cancer Res 16(23): 5805-5813.

Kao SC, van Zandwijk N, Clarke S (2014) Comment on 'Neutrophil to lymphocyte ratio in malignant pleural mesothelioma'. Br J Cancer 111(12): 2376.

Leonardo E, Zanconati F, Bonifacio D, Bonito LD (2001)

Immunohistochemical MIB-1 and p27kip1 as prognostic factors in pleural mesothelioma. Pathol Res Pract 197(4): 253-256.

Meert AP, Feoli F, Martin B, Verdebout JM, Mascaux C, Verhest A, Ninane V, Sculier JP (2004) Ki67 expression in bronchial preneoplastic lesions and carcinoma in situ defined according to the new 1999 WHO/IASLC criteria: a preliminary study. Histopathology 44(1): 47-53.

Meniawy TM, Creaney J, Lake RA, Nowak AK (2013) Existing models, but not neutrophil-to-lymphocyte ratio, are prognostic in malignant mesothelioma. Br J Cancer 109(7): 1813-1820.

Meniawy TM, Creaney J, Lake RA, Nowak AK (2014) Reply to Comment on 'Existing prognostic models, but not neutrophil-to-lymphocyte ratio, are prognostic in malignant mesothelioma'. Br J Cancer 111(12): 2377.

Pillai K, Pourgholami MH, Chua TC, Morris DL (2013) Ki67-BCL2 index in prognosis of malignant peritoneal mesothelioma. Am J Cancer Res 3(4): 411-423.

Rusch VW (1995) A proposed new international TNM staging system for malignant pleural mesothelioma. From the International Mesothelioma Interest Group. Chest 108(4): 1122-1128.

Sandeck HP, Roe OD, Kjaerheim K, Willen H, Larsson E (2010) Re-evaluation of histological diagnoses of malignant mesothelioma by immunohistochemistry. Diagn Pathol 5: 47.

Scagliotti GV, Micela M, Gubetta L, Leonardo E, Cappia S, Borasio P, Pozzi E (1993) Prognostic significance of Ki67 labelling in resected non small cell lung cancer. Eur J Cancer 29A(3): 363-365.

Scherpereel A, Astoul P, Baas P, Berghmans T, Clayson H, de Vuyst P, Dienemann H, Galateau-Salle F, Hennequin C, Hillerdal G, Le Pechoux C, Mutti L, Pairon JC, Stahel R, van Houtte P, van Meerbeeck J, Waller D, Weder W (2010) Guidelines of the European Respiratory Society and the European Society of Thoracic Surgeons for the management of malignant pleural mesothelioma. Eur Respir J 35(3): 479-495.

Schonherr A, Bayer M, Bocking A (2004) Diagnostic and prognostic value of Ki67 proliferation fraction in serous effusions. Cell Oncol 26(1-2): 57-62. 
Skov BG, Holm B, Erreboe A, Skov T, Mellemgaard A (2010) ERCC1 and Ki67 in small cell lung carcinoma and other neuroendocrine tumors of the lung: distribution and impact on survival. J Thorac Oncol 5(4): 453-459.

Stahel RA, Weder W, Lievens Y, Felip E (2010) Malignant pleural mesothelioma: ESMO Clinical Practice Guidelines for diagnosis, treatment and follow-up. Ann Oncol 21(Suppl 5): v126-v128.

Sugarbaker DJ, Wolf AS (2010) Surgery for malignant pleural mesothelioma. Expert Rev Respir Med 4(3): 363-372.

Tanrikulu AC, Abakay A, Kaplan MA, Kucukoner M, Palanci Y, Evliyaoglu O, Sezgi C, Sen H, Carkanat AI, Kirbas G (2010) A clinical, radiographic and laboratory evaluation of prognostic factors in 363 patients with malignant pleural mesothelioma. Respiration 80(6): 480-487.

Tsao AS, Wistuba I, Roth JA, Kindler HL (2009) Malignant pleural mesothelioma. J Clin Oncol 27(12): 2081-2090. van Zandwijk N, Clarke C, Henderson D, Musk AW, Fong K, Nowak A, Loneragan R, McCaughan B, Boyer M, Feigen M, Currow D, Schofield P, Nick Pavlakis BI, McLean J, Marshall H, Leong S, Keena V, Penman A (2013) Guidelines for the diagnosis and treatment of malignant pleural mesothelioma. J Thorac Dis 5(6): E254-E307.

Yerushalmi R, Woods R, Ravdin PM, Hayes MM, Gelmon KA (2010) Ki67 in breast cancer: prognostic and predictive potential. Lancet Oncol 11(2): 174-183.

This work is published under the standard license to publish agreement. After 12 months the work will become freely available and the license terms will switch to a Creative Commons AttributionNonCommercial-Share Alike 4.0 Unported License. 